\title{
Band Crossing Evidence in PbSnTe Observed by Optical Transmission Measurements
}

\author{
S. O. Ferreira ${ }^{1}$, E. Abramof ${ }^{2}$, P. Motisuke $^{2}$, P. H. O. Rappl ${ }^{2}$, H. Closs ${ }^{2}$, \\ A. Y. Ueta ${ }^{2}$, C. Boschetti ${ }^{2}$, and I. N. Bandeira ${ }^{2}$, \\ 1: Dep. Física, Universidade Federal de Viçosa, \\ 36571-000, Viçosa, MG, Brazil \\ 2: Instituto Nacional de Pesquisas Espaciais, \\ C.P. 515 - 12201-970 São José dos Campos, SP, Brazil
}

Received February 8, 1999

\begin{abstract}
Using high quality epitaxial layers, we have obtained direct evidence of the band inversion in the $P b_{1-x} S n_{x} T e$ system. The samples, covering the whole composition range, were grown by molecular beam epitaxy on (111) $B a F_{2}$ substrates. A minimum in the resistivity as a function of temperature was observed for all samples with $S n$ composition $0.35 \leq x \leq 0.70$. In the same samples and at the same temperature, temperature dependent optical transmission measurements have revealed a change in signal of the energy gap temperature derivative, a direct evidence of the band inversion. However, the temperature for which the inversion occurs is not the one expected by the band inversion model. This discrepancy is supposed to be due to the Burstein-Moss shift caused by the relatively high hole concentration observed in these samples.
\end{abstract}

\section{Introduction}

Lead-tin telluride is a narrow gap semiconductor which have been investigated for many years and applied mainly in the fabrication of infrared (3-14 $\mu \mathrm{m})$ photo detectors and diode lasers [1,2]. The growth of epitaxial layers and multi-layer structures like superlattices and multi-quantum wells of narrow gap $I V-V I$ compounds by molecular beam epitaxy (MBE) has improved the basic research of quantum effects in these materials [3,4]. New interesting research possibilities were opened by alloying the binary and also ternary lead salts with rare-earth elements (mainly $E u$ and $Y b$ ) to produce compounds with higher energy gaps and their respective multi-layer heterostructures [5]. Recently, the growth of lead salts on silicon substrates using fluoride buffer layers, in order to obtain the monolithic integration of lead salt detector arrays with silicon read-out circuits, has also received much attention $[6,7]$.

According to the band inversion (BI) model [8], the $P b_{1-x} S n_{x} T e$ energy gap $\left(E_{g}\right)$ initially decreases as the $S n$ composition increases, and vanishes for an intermediate alloy composition. Further increasing the $S n$ composition, the energy gap starts to increase, with the band edge states inverted, up to the $S n T e$ value. The $S n$ composition for which the band inversion should occur varies from $x \approx 0.35$ to $x \approx 0.70$ as the temperature increases from 4 to $300 \mathrm{~K}$. However, it is very difficult to determine the band edge structure near and beyond the band inversion region, since only high carrier concentration samples can be obtained due to the deviation from the stoichiometry [9]. The energy gap of $P b_{1-x} S n_{x} T e$ has been experimentally determined only for the range $x<0.25$. The Burstein-Moss shift, caused by the high hole concentration, which is observed in the samples with higher tin composition $(x>0.30)$, imposes difficulties in the determination of the "real" gap by optical-absorption measurements $[10,11]$. In this composition range, the only available $E_{g}$ experimental data has been determined by tunneling measurements in $\mathrm{Al}-\mathrm{Al}_{2} \mathrm{O}_{3}-\mathrm{SnTe}$ structures [12]. The "real" $E_{g}$ of pure $\operatorname{SnTe}\left(p \sim 10^{20} \mathrm{~cm}^{-3}\right)$ is $0.18 \mathrm{eV}$ [12], while the "optical energy gap" was found to be near $0.5 \mathrm{eV}$ $[10,11]$.

Using In doping to reduce carrier concentration, Takaoka et al. [13] have determined the effective masses and energy gap as a function of tin composition across the band-inversion region by the far-infrared magne- 
toplasma method. They have observed much heavier effective masses in these doped samples as compared with the values expected for the undoped $P b_{1-x} S n_{x} T e$ and an energy gap which does not go to zero. In their conclusions, they state that it is difficult to say if this behavior is essential for $P b S n T e$ or a result of the In doping. Although the BI model is widely accepted, direct observation for the $P b_{1-x} S n_{x}$ Te system has not been achieved yet, mainly due to the lack of good quality samples with $x>0.30$ and with low carrier concentration.

\section{Experimental}

Recently, we have reported on the growth of high quality $P b S n T e$ samples, covering the whole composition range [14]. The PbSnTe layers were grown on (111) $B a F_{2}$ substrates by molecular beam epitaxy using solid PbTe and SnTe sources. The growth temperature was between 250 and $300{ }^{\circ} \mathrm{C}$ and the final thickness was about $4 \mu \mathrm{m}$. The samples were characterized by high resolution x-ray diffraction, temperature dependent resistivity, Hall mobility and infrared transmission. The films grown using stoichiometric PbTe and SnTe sources have shown a hole concentration between $p=1 \times 10^{17} \mathrm{~cm}^{-3}$, for $P b_{0.85} S n_{0.15} T e$, to $p=2 \times 10^{19} \mathrm{~cm}^{-3}$, for $S n T e$. This $p$ value, observed for $S n T e$, is at least one order of magnitude lower than the one previously reported in the literature. Such a lower carrier concentration increases the hall mobilities of all samples and makes easier their optical characterization. Details about the electrical characterization of these layers have been published elsewhere [15].

Another important feature is a well defined minimum in the temperature dependent resistivity and a corresponding maximum in the mobility of all samples with $0.35 \leq x \leq 0.70$, as can be observed in the Fig. 1 for two typical $P b S n T e$ layers.

According to the $\mathrm{BI}$ model, this composition range corresponds to that where band crossing should occur. Since this behavior can be observed only for the samples in the band crossing range, it seems that this minimum in the resistivity is related to the band inversion. For the samples in this range, the energy gap should first reduce with decreasing temperature, as it happens for PbTe. But after the band inversion it should increase with further decrease in temperature, the behavior of SnTe. This change in the signal of the energy gap temperature coefficient $\left(d E_{g} / d T\right)$, would be the reason for the behavior observed in the resistivity and mobility.

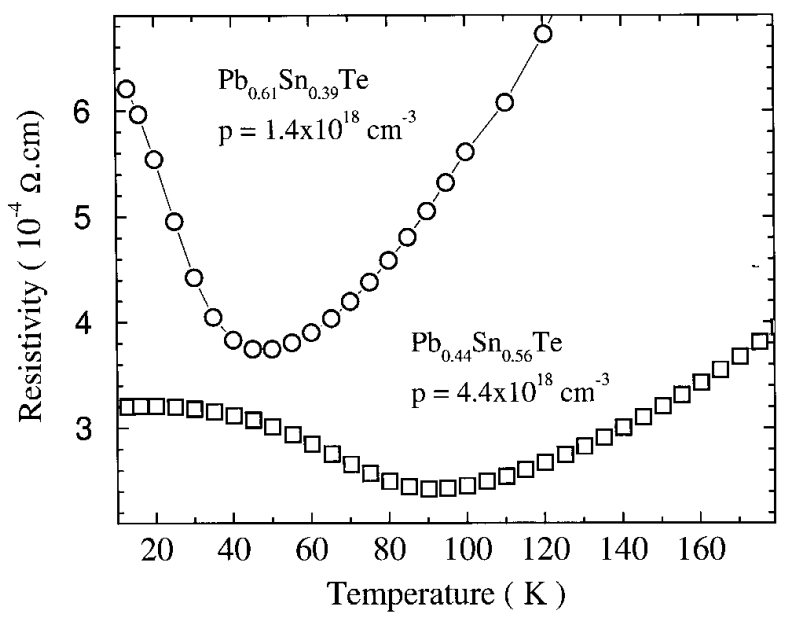

Figure 1. Resistivity as a function of temperature for two typical $P b S n T e$ samples in the band inversion region.

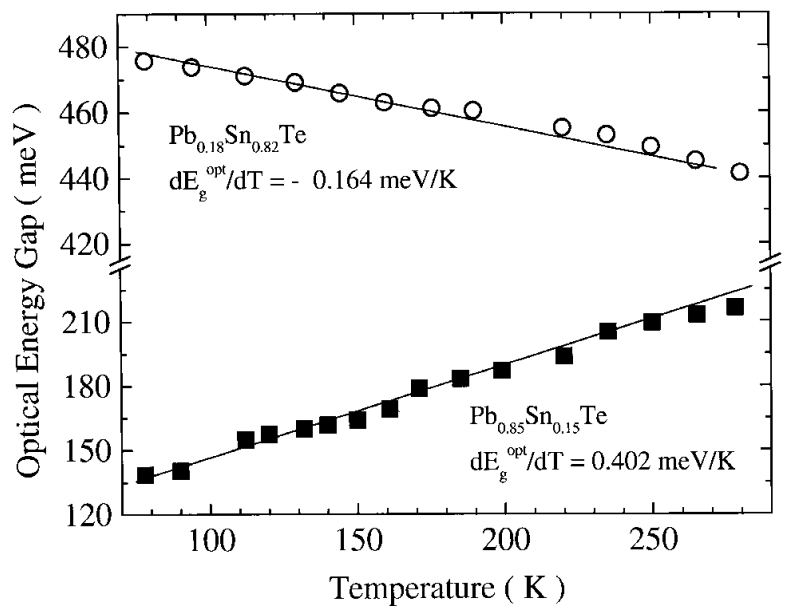

Figure 2. Optical energy gap as a function of temperature for two PbSnTe samples outside the BI region.

To clarify this point, the temperature dependence of the energy gap for all samples, mainly that covering the BI region, was measured. The energy gap was determined from the transmission spectra, which were measured using a Fourier transform infrared spectrometer in the range from 4500 to $800 \mathrm{~cm}^{-1}$ for temperatures between 5 and $300 \mathrm{~K}$. Fig. 2 shows the energy gap as a function of temperature for two samples outside the BI region. 


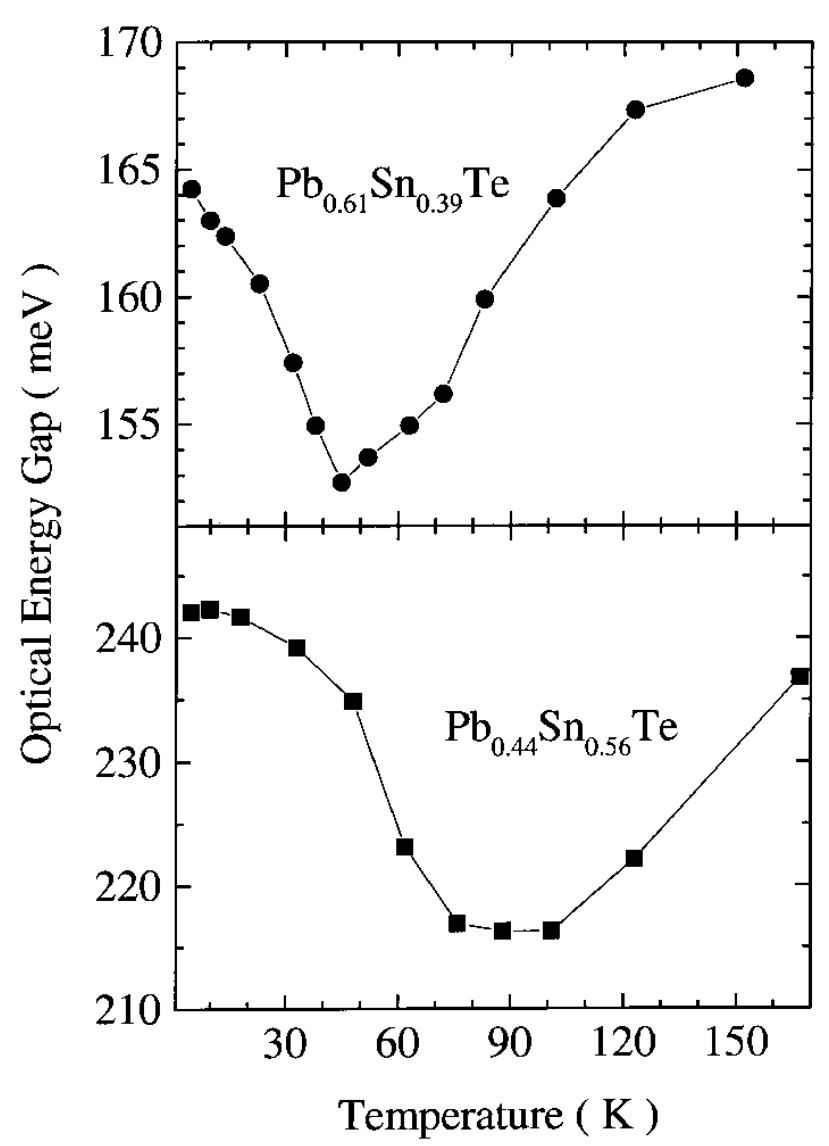

Figure 3. Optical energy gap as a function of temperature for two typical PbSnTe samples in the BI region. The lines are guides to the eye.

As expected, the energy gap increases with temperature, for the sample with $x=0.15$, while it decreases, for the sample with $x=0.82$. But, in contrast to model, the absolute value of the temperature coefficients $\left(d E_{g} / d T\right)$ were very different. This occurs because the $E_{g}$ value taken from the transmission spectra is the "optical energy gap" $\left(E_{g}^{o p}\right)$, which takes into account the Burstein-Moss (BM) shift, due to the band filling. For the $P b_{0.85} S n_{0.15}$ Te layer the $\mathrm{BM}$ shift is negligible $\left(p=3 \times 10^{17} \mathrm{~cm}^{-3}\right)$ and the measured values of $E_{g}$ and $d E_{g} / d T$ are the ones expected from the literature [16]. On the other hand, for the sample with $x=0.82\left(p=1 \times 10^{19} \mathrm{~cm}^{-3}\right)$, the BM shift is 230 meV at $80 \mathrm{~K}$, and $d E_{g}^{o p} / d T$ is a combination of the changes in the energy gap and in the position of the Fermi level. Preliminary calculations of the absorption coefficient, using a model proposed by Anderson [17], which takes the BM shift into account, explain the reduction in $d E_{g}^{o p} / d T$ observed for samples with high hole concentration.

The temperature dependence of $E_{g}^{o p}$ for the samples in the band inversion region is completely different, as shown Fig. 3.

The optical energy gap first reduces, reaches a minimum, and than starts to increase. Again, due to the relatively high carrier concentration of the samples, the temperature coefficient of $E_{g}^{o p}$ is much smaller than the expected value of $d E_{g} / d T$. The temperature where this minimum in $E_{g}^{o p}$ occurs coincides with the temperature of minimum resistivity for all samples in the band inversion region. Fig. 4 plots the temperature of minimum resistivity and minimum $E_{g}^{o p}$ as a function of $S n$ content for all our MBE samples, together with the band crossing temperature predicted from the BI model.

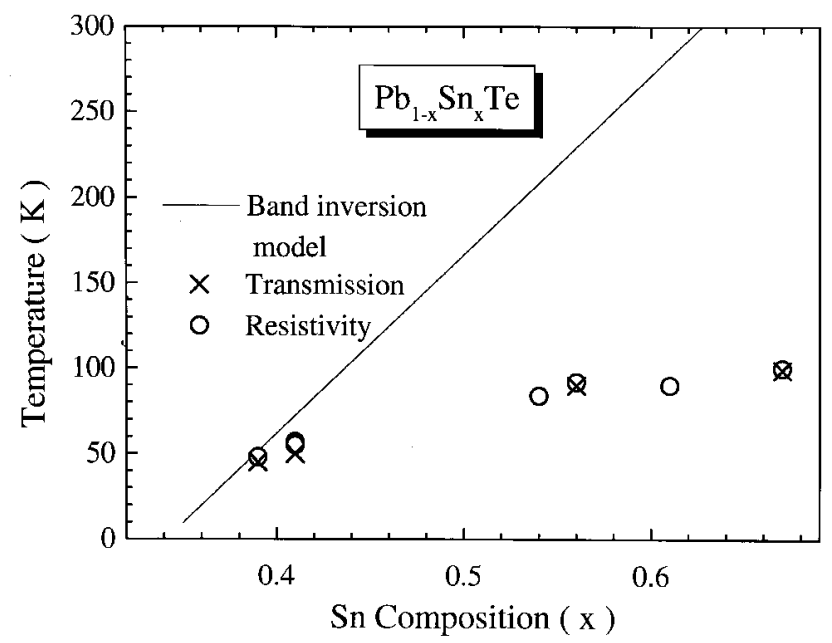

Figure 4. Temperatures of minimum resistivity $(0)$ and of $d E_{g}^{o p} / d T=0(\times)$. The solid line is the band crossing temperature calculated from the BI model.

\section{Conclusion}

The Burstein-Moss shift in the PbSnTe system, due to the high hole concentration, produces a strong change in the temperature dependence and absolute value of the optical energy gap. The change in signal of $d E_{g}^{o p} / d T$, for $P b_{1-x} S n_{x} T e$ samples with $0.35 \leq x \leq$ 0.70 , is a direct experimental evidence of the band inversion in this alloy. The coincidence between the optical and electrical measurements shown in Fig. 4, indicates that the minimum previously observed in the resistivity curves of our samples is related to the band crossing. The discrepancy between the measured and predicted temperatures, which can be observed in Fig. 4 is not yet clearly understood, but we believe that it is also related to the effect of high carrier concentration of these samples, since the BI model does not take in account the BM shift. 


\section{Acknolegments}

This work has been partially supported by "Conselho Nacional de Desenvolvimento Científico e Tecnológico-CNPq" and "Fundação de Amparo À Pesquisa do Estado de São Paulo-FAPESP".

\section{References}

1. H. Holloway and J.N. Walpole, Prog. Crystal Growth Charact. 2, 49 (1979).

2. H. Preier, Appl. Phys. 20, 189 (1979).

3. D.L. Partin, in: Semiconductors and Semimetals, Vol. 33, Ed. T.P. Pearsall (Academic Press, New York, 1991) p. 311.

4. G. Springholz, G. Ihninger, G. Bauer, M.M. Olver, J.Z. Pastalan, S. Romaine and B.B. Goldberg, Appl. Phys. Lett. 63, 2908 (1993).

5. G. Springholz and G. Bauer, J. Crystal Growth 144, 157 (1994).

6. C. Boschetti, P.H.O. Rappl, A.Y. Ueta and I.N. Bandeira, Infrared Phys. 34, 281 (1993).

7. H. Zogg, A. Fach, C. Maissen, J. Masek and S. Blunier, Optical Engineering 33, 1440 (1994).
8. J. Dimmock, I. Melngailis and A.J. Strauss, Phys. Rev. Lett. 16, 1193 (1966).

9. T. C. Harman, J. Nonmetals, 1, 183 (1973).

10. E.G. Bylander, J.R. Dixon, H.R. Riedl and R.B. Schoolar, Phys. Rev. 138, A873 (1965).

11. R.B. Schoolar, H.R. Riedl and J.R. Dixon, Solid State Commun. 4, 423 (1966).

12. L. Esaki and P.J. Stiles, Phys. Rev. Lett. 16, 1108 (1966).

13. S. Takaoka, S. Shimomura, H. Takahashi and K. Murase, Phys. Rev. B, 40, 5642 (1989)

14. P.H.O. Rappl, H. Closs, S.O. Ferreira, E. Abramof, C. Boschetti, P. Motisuke, A.Y. Ueta and I.N. Bandeira, J. Cryst. Growth, 191, 466 (1998).

15. E. Abramof, S.O. Ferreira, P.H.O. Rappl, H. Closs And I.N. Bandeira, J. Appl. Phys., 82, 2405 (1997).

16. G. Nimtz, in Numerical Data and Functional Relationships in Science and Technology, eds: K. H. Hellwege and O. Madelung, Landolt-Börstein, New Series Group III, vol. 17, Part F, p 170.

17. W. W. Anderson, Infrared Phys, 20, 363 (1980). 